\title{
On the Correlation Dimension of the Spectral Measure for the Thue-Morse Sequence
}

\author{
Michael A. Zaks, ${ }^{1}$ Arkady S. Pikovsky, ${ }^{1}$ and Jürgen Kurths ${ }^{1}$
}

Received February 11, 1997; final March 26, 1997

\begin{abstract}
Using the relationship between the decay rate of autocorrelation and the characteristics of singular Fourier spectra, we show that the correlation dimension of the spectral measure for the infinite Thue-Morse symbolic sequence equals $3-\log (1+\sqrt{17}) / \log 2=0.64298 \ldots$
\end{abstract}

KEY WORDS: Singular continuous spectrum; multifractality; autocorrelation function.

The Thue-Morse infinite binary sequence $\left\{M_{j}\right\}(j=1,2, \ldots)$ is formed by two symbols (in our notation, 1 and -1 ) and can be obtained from the starting point $M_{1}=1$ by means of repetitive substitutions (inflations) according to the rule ${ }^{(1,2)}$

$$
\begin{array}{rrrr}
1 & \rightarrow & 1 & -1 \\
-1 & \rightarrow & -1 & 1
\end{array}
$$

Several equivalent definitions are possible; for instance, $M_{j}=(-1)^{k+1}$, where $k$ is the sum of digits in the binary representation of $j$. With respect to a shift along the symbolic chain, the Thue-Morse sequence supports a unique translation-invariant ergodic probability measure. ${ }^{(3)}$ Although organized by a simple deterministic rule, the Thue-Morse sequence is not periodic. The Fourier spectrum (in physical applications the term "structure factor" is frequently used) of the infinite chain has long been known to be purely singular continuous: ${ }^{(4,5)}$ the spectral measure is neither atomic (discrete) nor absolutely continuous with respect to the Lebesgue measure,

\footnotetext{
${ }^{1}$ Department of Nonlinear Dynamics, Institute of Theoretical Physics and Astrophysics, Potsdam University, Postfach 601553, D-14415 Potsdam, Germany.
} 
but is concentrated on a Cantor set. This convenient combination of computational simplicity and intrinsic complexity has turned the Thue-Morse sequence into a standard tool, widely exploited in the context of long-range ordering and disordering in one-dimensional patterns. To mention only some recent applications, it was prescribed as a governing law for the external force in a kicked quantum rotator ${ }^{(6,7)}$ and served as a groundstate configuration for the classical lattice gas model ${ }^{(8)}$ and Ising spin system with short-range interactions. ${ }^{(9)}$ In the description of the critical phenomena for the aperiodic quantum Ising lattices ${ }^{\left(10 .{ }^{12)}\right.}$ it dictated the coupling constants. Further, the Thue-Morse sequence played the role of a one-dimensional potential for the discretized Schrödinger equation in the study of the gap distribution in the energy spectrum, ${ }^{(13)}$ and its singular spectra have been recovered in the diffraction patterns of specially prepared GaAs-AlAs superlattice heterostructures; ${ }^{(14,15)}$ the latest advances in this direction are thoroughly discussed in ref. 16 (where also an extensive list of references can be found). Finally, the Thue-Morse symbolic code of the attractor at the accumulation point of the sequence of "homoclinic doublings" in symmetric flows with saddle equilibria ${ }^{(17,18)}$ was recently understood as one of the mechanisms responsible for the generation of singular continuous spectra in continuous dissipative systems. ${ }^{(19)}$

The multifractal properties of the Fourier spectrum for the ThueMorse sequence have been investigated by Godrèche and Luck, ${ }^{(20)}$ who determined numerically the generalized dimensions $D_{q}$ and the singularity spectrum $f(\alpha)$ for the spectral measure (see also a recent application of the wavelet technique in ref. 21). Apparently, the box-counting dimension $D_{0}$ equals 1 (the Cantor set which carries the spectral measure is dense); estimation of $D_{q}$ for the general case $q \neq 0$ requires extensive numerical computations.

In this note we explicitly calculate the value of the correlation dimension $D_{2}$ for the spectral measure. In doing this, we utilize the exact relation derived in refs. 22-24, which connects the properties of the autocorrelations to those of the singular spectral measure, and can be formulated as follows. Let the autocorrelation function for an observable $x_{j}(j=1,2, \ldots)$ be introduced as $C(t)=\left(\left\langle x_{j} x_{j+t}\right\rangle-\left\langle x_{j}\right\rangle^{2}\right) /\left(\left\langle x_{j}^{2}\right\rangle-\left\langle x_{j}\right\rangle^{2}\right)$ and the integrated autocorrelation function as $C_{\text {int }}(T)=(1 / T) \sum_{t=0}^{T}|C(t)|^{2}$, respectively. Then $C_{\text {int }}(T) \sim T^{-D_{2}}$, where $D_{2}$ is the correlation dimension of the spectral measure. Thus the direct evaluation of $D_{2}$ from the Fourier spectral data can be replaced by the estimation of the decay rate for the integrated autocorrelation; as shown below, the self-similarity of the Thue-Morse sequence allows us to determine this rate analytically (note that the autocorrelation function generically does not depend on the choice of observable, so we simply put $x_{j}=M_{j}$ ). 
By construction the Thue-Morse sequence is invariant under the substitution (1) and under the inverse operation of "binary decimation": crossing out each even symbol. This is reflected in the recurrence properties of the correlation function: $\sum_{j} M_{j} M_{j+t}=\sum_{j} M_{j} M_{j+2 t}$ and, respectively,

$$
C(2 t)=C(t)
$$

This ensures repetitive nondecaying peaks for arbitrarily large values of $t$ (and thus prohibits the spectrum from being absolutely continuous with respect to the Lebesgue measure: the necessary condition of such continuity is the decay of correlations for $t \rightarrow \infty))^{(25,26)}$ The analogous recurrence relation for the odd argument values follows from the invariance of the infinite sum $\sum_{j} M_{j} M_{j+2 t+1}$ under the binary decimation. After decimation, the odd symbols remain unchanged, whereas the even ones are replaced by their counterparts ( 1 by -1 and vice versa), hence the pairwise products acquire the opposite sign. Consequently,

$$
C(2 t+1)=-\frac{C(t)+C(t+1)}{2}
$$

The relations (2), (3) combined with the "initial condition" $C(0)=1$ allow one to obtain the exact values of $C(t)$ for any value of $t$. They ensure that the inequality $|C(t)| \leqslant 1 / 3$ holds for all $t$ [naturally, except for $C(0)=1$ ].

Consider now the recurrences between the sums $S_{n} \equiv \sum_{n+1}^{2 n} C^{2}(t)$ for $n=m, 2 m, 4 m$,..., with $m$ an arbitrary positive integer. On applying (2) and (3) to the even and odd terms in $S_{4 m}$, respectively, we obtain

$$
S_{4 m}=S_{2 m}+\frac{S_{2 m}}{2}+\frac{1}{2} \sum_{2 m}^{4 m-1} C(t) C(t+1)
$$

Denoting the last term in the right hand side of (4) by $\Pi_{2 m}$ and transforming it with the help of (2), (3), we arrive at

$$
-\Pi_{2 m}=-\sum_{m}^{2 m-1}\left(C^{2}(t)+C(t) C(t+1)\right)
$$

Thus we are left with the two coupled linear recurrences

$$
\begin{aligned}
S_{2 j} & =\frac{3}{2} S_{j}+\frac{1}{2} \Pi_{j} \\
\Pi_{2 j} & =-S_{j}-\Pi_{j}
\end{aligned}
$$


Introducing further the variables $\kappa_{j} \equiv S_{2 j} / S_{j}$ and $\xi_{j} \equiv \Pi_{2 j} / S_{j}$ and noticing that the equality $\kappa_{j}\left(\xi_{j}+2\right)=2$ is independent of $j$, we end up with a simple one-dimensional mapping

$$
\kappa_{2 j}=\frac{1}{\kappa_{j}}+\frac{1}{2}
$$

which in the domain $\kappa>0$ (only this is of relevance, since $S_{j}>0$ ) has the globally attracting fixed point $\kappa_{*}=(1+\sqrt{17}) / 4=1.280776406 \ldots$. Therefore, for large $j$, we have $S_{m 2^{\prime}} \propto\left(\kappa_{*}\right)^{j} S_{m}$. Let us turn now to the integrated autocorrelation $C_{\text {int }}(T)$. Since this function is by definition a ratio of two monotonic functions of $T$, it is easy to see that, irrespective of $T$, the inequality

$$
\frac{C_{\text {int }}(2 T)}{2}<C_{\text {int }}(t)<2 C_{\text {int }}(T)
$$

holds for any $t$ from the interval $(T, 2 T)$. Obviously, for the values $T=2^{n} m$ under large $n$,

$$
C_{\mathrm{int}}(T)=\frac{1+\sum_{j=1}^{n}-S_{m 2^{j}}}{m 2^{n}} \propto T^{\left(\log \kappa_{*} / \log 2\right)-1}
$$

In combination with (8), this ensures the power-law decay of $C_{\text {int }}(T)$. According to Wiener, ${ }^{(25)}$ vanishing of $C_{\text {int }}(\infty)$ implies the absence of the discrete component in the spectrum. Consequently, the spectral measure for the Thue-Morse sequence is purely singular continuous, and its correlation dimension equals

$$
D_{2}=1-\frac{\log \kappa_{*}}{\log 2}=3-\frac{\log (1+\sqrt{17})}{\log 2}=0.64298136 \ldots
$$

Compare this with the values of other generalized dimensions: the capacity $D_{0}=1$ and the information dimension $D_{1}=0.730$ (numerical value).

The fact that the relation (9) is independent of $m$ implies that the prefactor before $T^{1-D_{2}}$ on the right-hand side of (9) must tend to a periodic function of the $\log$ arithmic variable $\log T / \log 2$. Our numerical estimates, however, show the influence of this time dependence to be rather weak: the prefactor oscillates $\log$-periodically between 0.75 and 0.77 .

The same procedure can be implemented for other symmetrical substitution sequences, for instance, for the $m$-tuplings: $A \rightarrow A B^{m-1}$, 
$B \rightarrow B A^{m-1}$. Obviously, the case $m=2$ yields the Thue-Morse sequence. For $m=3$ the autocorrelation function obeys the recurrences

$$
C(3 t)=C(t), \quad C(3 t+1)=-\frac{C(t+1)}{3}, \quad C(3 t+2)=-\frac{C(t)}{3}
$$

and the correlation dimension of the spectral measure equals $3-\log 11 /$ $\log 3=0.8173 \ldots$. For $m=4$ one has

$$
C(4 t)=C(t), \quad C(4 t+1)=-C(4 t+3)=\frac{C(t)-C(t+1)}{4}, \quad C(4 t+2)=0
$$

and

$$
D_{2}=\frac{5}{2}-\frac{\log (3+\sqrt{41})}{\log 4}=0.8834 \ldots
$$

Higher values of $m$ can be treated in a similar way.

We expect that knowledge of the recurrent properties for the autocorrelation function of substitution symbolic sequences [the obvious relation (2) is commonly used, but less evident relations like (3) seem to be overlooked] and that of the exact values like (10) can be helpful in answering many subtle questions which arise in the context of one-dimensional "quasicrystals" in substitutional systems with various bond-length ratios, ${ }^{(27,28)}$ extended electronic states in one-dimensional lattices, ${ }^{(29)}$ and kicked quantum systems with corresponding potentials. ${ }^{(6,7)}$

\section{REFERENCES}

1. A. Thue, Über unendliche Zeichenreihen, Norske Vid. Selsk. Skr. I Mat. Nat. Kl. Christiania 7:1-22 (1906).

2. M. Morse, Recurrent geodesics on a surface of negative curvature, Trans. A.M.S. 22:84-100 (1921).

3. M. Keane, Generalised Morse sequences, Z. Wahrsch. 10:335-353 (1968).

4. K. Mahler, On the translation properties of a simple class of arithmetical functions, J. Math. Phys. 6:158-163 (1926).

5. M. Queffélec, Substitution Dynamical Systems-Spectral Analysis (Springer, Berlin, 1987).

6. M. Combescure, Recurrent versus diffusive dynamics for a kicked quantum system, J. Stat. Phys. 62:779-791 (1991).

7. C. R. de Oliveira, Numerical study of the long-time behaviour of quantum systems driven by Thue-Morse forces. Application to two-level systems, Europhys. Lett. 31:63-68 (1995).

8. A. van Enter and J. Miękisz, Breaking of periodicity at positive temperatures, Commun. Math. Phys. 134:647-651 (1990).

9. C. Gardner, J. Miękisz, C. Radin, and A. van Enter, Fractal symmetry in an Ising model, J. Phys. A 22:L1019-L1023 (1989). 
10. J. M. Luck, Critical behavior of the aperiodic quantum Ising chain in a transverse magnetic field, J. Stat. Phys. 72:417-458 (1993).

11. L. Turban, F. Igloi, and B. Berche, Surface magnetization and critical behavior of aperiodic Ising quantum chains, Phys. Rev. B 49:12695-12702 (1994).

12. F. Igloi and P. Lajko, Surface magnetization and surface correlations in aperiodic Ising models, J. Phys. A: Math. Gen. 29:4803-4814 (1996).

13. Z. Cheng, R. Savit, and R. Merlin, Structure and electronic properties of Thue-Morse lattices, Phys. Rev. B 37:4375-4382 (1988).

14. F. Axel and H. Terauchi, High-resolution X-ray-diffraction spectra of Thue-Morse GaAs-AlAs heterostructures: Towards a novel description of disorder, Phys. Rev. Lett. 66:2223-2226 (1991).

15. J. Peyrière, E. Cockayne, and F. Axel, Spectra of finite-size Thue-Morse GaAs-AlAs multilayer heterostructures, J. Phys. France I 5:111-128 (1995).

16. A. Bovier and J.-M. Ghez, Remarks on the spectral properties of tight-binding and Kronig-Penney models with substitution sequences, J. Phys. A: Math. Gen. 28:2313-2324 (1995).

17. A. Arneodo, P. Coullet, and C. Tresser, A possible new mechanism for the onset of turbulence, Phys. Lett. A 81:197-201 (1981).

18. D. V. Lyubimov and M. A. Zaks, Two mechanisms of the transition to chaos in finitedimensional models of convection, Physica D 9:52-64 (1983).

19. A. S. Pikovsky, M. A. Zaks, U. Feudel, and J. Kurths, Singular continuous spectra in dissipative dynamical systems, Phys. Rev. E 52:285-296 (1995).

20. C. Godrèche and J. M. Luck, Multifractal analysis in reciprocal space and the nature of the Fourier transform of self-similar structures, J. Phys. A: Math. Gen. 23:3769-3797 (1990).

21. E. Liviotti, A study of the structure factor of Thue-Morse and period-doubling chains by wavelet analysis, J. Phys. Condensed Matter 8:5007-5015 (1996).

22. R. Ketzmerick, G. Petschel, and T. Geisel, Slow decay of temporal correlations in quantum systems with Cantor spectra, Phys. Rev. Lett. 69:695-698 (1992).

23. M. Holschneider, Fractal wavelet dimensions and localization, Common. Math. Phys. 160:457-473 (1994).

24. C.-A. Guerin and $M$. Holschneider, On equivalent definitions of the correlation dimension for a probability measure, $J$. Stat. Phys. 86:707-720 (1997).

25. N. Wiener, The spectrum of an array, J. Math. Phys. 6:145-157 (1926).

26. I. P. Cornfeld, S. V. Fomin, and Ya. G. Sinai, Ergodic Theory (Springer, New York, 1982).

27. M. Kolar, New class of one-dimensional quasicrystals, Phys. Rev. B 26:5489-5492 (1993).

28. M. Kolar, B. lochum, and L. Raymond, Structure factor of 1D systems (superlattices) based on two-letter substitution rules, J. Phys. A: Math. Gen. 26:7343-7366 (1993).

29. A. Chakrabarti, S. N. Karmakar, and R. K. Moitra, Role of a new type of correlated disorder in extended electronic states in the Thue-Morse lattice, Phys. Rev. Lett. 74:1403-1406 (1995). 